\title{
Tying up loose ends: telomeres, genomic instability and lamins
}

\author{
Susana Gonzalo ${ }^{a^{*}} \&$ Joel C. Eissenberg ${ }^{a}$
}

${ }^{a}$ Edward A. Doisy Department of Biochemistry and Molecular Biology

Saint Louis University School of Medicine

Doisy Research Center

1100 South Grand Blvd.

St. Louis, MO 63104, USA

Email (JCE): eissenjc@slu.edu

Email (SG): sgonzalo@slu.edu

${ }^{*}$ Corresponding author

(C) 2016. This manuscript version is made available under the Elsevier user license http://www.elsevier.com/open-access/userlicense/1.0/ 


\section{Abstract}

On casual inspection, the eukaryotic nucleus is a deceptively simple organelle. Far from being a bag of chromatin, the nucleus is, in some ways, a structural and functional extension of the chromosomes it contains. Recently, interest has intensified in how chromosome compartmentalization and dynamics affect nuclear function. Different studies uncovered functional interactions between chromosomes and the filamentous nuclear meshwork comprised of lamin proteins. Here, we summarize recent research suggesting that telomeres, the capping structures that protect chromosome ends, are stabilized by lamin-binding and that alterations in nuclear lamins lead to defects in telomere compartmentalization, homeostasis and function. Telomere dysfunction contributes to the genomic instability that characterizes agingrelated diseases, and might be an important factor in the pathophysiology of lamin-related diseases.

\section{Introduction}

Telomeres are specialized structures at the ends of linear eukaryotic chromosomes that mask the DNA ends from normal mechanisms sensing DNA breaks. This masking prevents telomeres from fusing with de novo breaks or with each other, and from triggering the normal cellular responses to DNA breaks: cell cycle arrest and, in the absence of repair, apoptosis. During replication, telomeres pose a challenge to chromosome maintenance: how is telomeric DNA on the lagging strand fully replicated? The answer is that conventional DNA replication is supplemented by the addition of telomeric DNA by the telomerase enzyme complex[1]. However, telomerase is expressed at low levels in somatic cells. Thus, telomere length progressively shortens during aging in most cells, contributing to the genomic instability that characterizes aging-related diseases. Significantly, tumor cells stabilize their telomeres, either 
by upregulating telomerase or by activating an alternative lengthening of telomeres (ALT) mechanism[2].

In addition to telomerase, a plethora of factors regulate telomere homeostasis. The discovery and characterization of a telomere-specific proteinaceous complex known as shelterin, telomere-binding DNA repair factors, non-coding RNAs specific for telomeres (TERRAs), and telomeric epigenetic modifications, have improved our understanding of telomere biology. Many reviews describe how these factors regulate telomere stability and function[2-4]. Interest in the field has recently focused on understanding how the compartmentalization and dynamics of telomeres in the nucleus affect telomere function. This review focuses on the emerging role of lamins, structural components of the nuclear lamina and the nucleoplasm, in the compartmentalization and dynamics of telomeres in the nucleus, and in the maintenance of telomere homeostasis and function. Lamin mutations, overexpression, or loss are associated with numerous degenerative disorders and premature aging syndromes. Uncovering how lamins regulate telomere biology will improve our knowledge of the pathophysiology of lamin-related diseases.

\section{Telomere structure}

Telomeres are nucleoprotein structures consisting of a long array of DNA repeats of the TTAGGG sequence (in vertebrates), which acquire a specialized higher-order lariat structure known as the "T-loop" (Figure 1). The formation and stabilization of this structure requires a minimal length of telomeric repeats, and is facilitated by a protein complex named "shelterin"[2,3]. The shelterin complex consists of six proteins: TRF1, TRF2, RAP1, TIN2, TPP1 and POT1 that bind specifically telomeric repeats (Figure 1). In this "capped" conformation, chromosome ends are physically protected from cellular activities that erode DNA or from being recognized as breaks by the DNA repair machinery. Telomere shortening below a critical 
threshold, or loss of shelterin complex components impairs T-loop formation. These uncapped telomeres are considered damaged or dysfunctional, as they are prone to erosion by nucleases and are also aberrantly "fixed" by fusion with other chromosome ends. Damaged/dysfunctional telomeres are a major cause of genomic instability in aging and cancer.

Telomere homeostasis is primarily maintained by telomerase, consisting of a reverse transcriptase catalytic subunit (TERT), an RNA component (TERC), and accessory factors[1]. Telomeres are also enriched in histone marks characteristic of repressed heterochromatin, which is important for maintaining telomere length homeostasis[5,6]. Telomere length is also regulated by DNA methylation of the neighboring subtelomeric regions[7]. Although telomeric TTAGGG repeats do not contain the CpG sequence that is susceptible to methylation by DNA methyltransferases (DNMTs), adjacent subtelomeric regions have high density of methylated CpG sequences. Loss of function of DNMTs results in reduced methylation of subtelomeric domains and in a marked increase in average telomere length. This telomere elongation seems to be due to increased recombination[7]. Despite their heterochromatic nature, telomeres are transcribed by RNA polymerase II, generating long non-coding RNAs. The telomeric repeatcontaining RNAs (TERRAs) initiate sub-telomerically, and have sequence complementarity with the telomerase template RNA. TERRA promoter regions have been mapped to CpG islands in proximity to telomere repeats tracts, and also to regions located 5-10 kilobases proximal to the telomeric repeats. TERRAs serve as scaffolds to recruit proteins to telomeres, regulating telomerase activity, heterochromatin formation and the DNA damage response triggered by dysfunctional telomeres[4]. Recently, TERRAs have also been found to co-purify with exosome vesicles. These extracellular TERRAs have been implicated in inflammatory cytokine stimulation, suggesting a role in immune signaling[8].

\section{Telomere compartmentalization and dynamics}


Genomic regions are compartmentalized into spatial domains within the nucleus[9]. The implications of nuclear compartmentalization for genome function are the focus of intensive research. Factors considered important for nuclear compartmentalization include chromatin modifications and association of chromatin with the nuclear envelope and/or nuclear matrix[10,11]. As such, heterochromatin domains accumulate at the nuclear periphery and surrounding nucleoli, while euchromatin extends throughout the nuclear interior[12].

Telomeres are heterochromatic domains with preferential locations near the nuclear envelope in proliferating yeast, mouse lymphocytes and mouse embryonic fibroblasts[13]. In human cells, telomeres show a scattered distribution throughout the entire nucleus, although perinucleolar and peripheral localization of specific telomeres has been reported[14]. In particular, specific subtelomeric sequences can direct peripheral localization of the corresponding telomere, suggesting that telomeres of different chromosomes might have preferred nuclear locations. In quiescent cells, telomere positioning within the nucleus is sensitive to metabolic status, which may contribute to cell longevity[15]. Furthermore, the transformed state may impact telomeric associations; the telomeres in a mammary epithelial cell line were found to be closer together than the same telomeres in a breast tumor cell line[16]. Although further studies are needed to determine how telomere compartmentalization impacts telomere homeostasis and function, there is evidence that the nuclear positioning of telomeres is altered in cellular contexts of telomere deregulation, such as in the case of senescence and immortality.

Telomeres are also dynamic structures, exhibiting highly variable non-directional motion. In meiotic cells, telomere-led rapid chromosome movement during meiotic prophase, mediated by microtubules and dynein, are believed to contribute to the establishment of homologous pairing and crossing over[17]. In cancer cells, telomere motion is inversely proportional to telomere length and independent of distance from the nuclear periphery[18]. A recent study also 
implicated cell geometry and forces from the cytoskeleton/nucleoskeleton in telomere motion[19]. In particular, culturing fibroblasts on substrates that emulate different extracellular matrix constrains resulted in dramatically different telomere mobility. A model was proposed whereby extracellular matrix signals change cytoskeletal organization and lamins expression, which in turn impact telomere/chromatin movement.

Interestingly, dysfunctional telomeres that result from excessive attrition of telomeric DNA or loss of the capping structure are more mobile and sample larger nuclear territories than functional telomeres[18,20]. The increased mobility is dependent upon ATM activation and expression of 53BP1, a key factor in DNA double-strand break (DSB) repair by non-homologous end joining (NHEJ)[20]. A model was proposed whereby 53BP1 binding to DNA double-strand breaks (DSBs) or dysfunctional telomeres changes the dynamic behavior of the surrounding chromatin, promoting the joining of broken DNA located at distant sites. Consistent with this notion, 53BP1 is required for the aberrant processing of dysfunctional telomeres by NHEJ, which results in chromosome end-to-end fusions. To date, the molecular mechanisms behind the proposed changes in chromatin dynamic behavior by 53BP1 binding remain unknown.

\section{Role of lamins in telomere homeostasis}

Nuclear lamins (A-type and B-type) are intermediate filaments that together with laminassociated proteins form a meshwork under the inner nuclear membrane known as the nuclear lamina (Figure 2). The ratios of A-type and B-type lamins are tightly regulated in different cells and tissues, contributing to differences in nuclear stiffness and mechanical stability among the tissues [21]. Lamin B provides elasticity to the nucleus and maintains its shape, while lamin A provides the viscosity that impedes nuclear deformation. Lamin A is more mobile than lamin B and forms a meshwork that extends throughout the nucleoplasm. Lamins are considered a scaffold for the organization of nuclear function[9,22]. Accordingly, alterations in lamins are 
associated with defects in nuclear shape, organization of chromatin and nuclear pore complexes, nuclear mechanical stress, as well as deficiencies in DNA replication, transcription, and repair[22,23]. A-type lamins (lamin $\mathrm{A}$ and $\mathrm{C}$ ) result from alternative splicing of the LMNA gene. Lamin A is synthesized as a prelamin A precursor that undergoes post-translational processing to produce mature lamin A, including farnesylation, carboxymethylation and proteolytic cleavage. Abnormal accumulation of prelamin A results in nuclear shape abnormalities and premature senescence in primary endothelial cells from cord blood or umbilical vein[24]. Mutant lamin A also leads to nuclear abnormalities and impaired mitosis, eventually leading to senescence[25].

Over 400 mutations in the LMNA gene have been associated with over a dozen degenerative disorders, including muscular dystrophies and premature aging diseases[26]. Hutchinson Gilford Progeria Syndrome (HGPS) and Restrictive Dermopathy (RD) are the most severe progeroid laminopathies. $\mathrm{RD}$ is a lethal neonatal laminopathy caused by mutation in either LMNA or Zmpste24 (FACE-1), the enzyme that processes prelamin A into mature lamin A. HGPS is caused by a mutation in the LMNA gene that activates a cryptic splice site, leading to the expression of a truncated protein known as progerin, which causes cellular toxicity[27]. HGPS patients exhibit growth impairment and numerous premature aging characteristics, dying in their teens from cardiovascular complications. Importantly, progerin is also expressed in cells from old individuals, causing nuclear alterations that recapitulate the phenotype of HGPS cells[28]. Thus, progerin accumulation is also thought to contribute to cellular degeneration during physiological aging. Understanding progerin regulation and its pathological activity is the focus of extensive current research.

Studies using cells derived from human patients and from mouse models of laminopathies show that defects in DNA repair and telomere maintenance, epigenetic alterations, and oxidative stress all contribute to genomic instability in laminopathies. Several recent reviews 
discuss molecular mechanisms behind this genomic instability[22,29,30]. Here, we discuss recent advances in understanding the molecular mechanisms behind telomere dysfunction in laminopathies.

Shoeman and Traub[31] first reported in vitro DNA-binding properties of purified nuclear lamins. In particular, lamin $\mathrm{A} / \mathrm{C}$ bound a synthetic oligonucleotide containing human telomeric repeats. Further studies showed that lamins and lamin-associated proteins such as LAP2 $\alpha \quad \square \square \square \square \square \square$ associate with telomeres in vivo[13,32,33] (Figure 2). 3D time-lapse live cell imaging showed that LAP2 $\alpha$ is mostly found at the nucleoplasm in interphase, in the cytoplasm in metaphase, and associating with telomeres in anaphase, before it relocates to the nuclear interior in late telophase [32]. It is not clear if the transient association of LAP2 $\alpha$ with telomeres is mediated by telomeric proteins or by direct binding to DNA. LAP2 $\alpha$ also forms a complex in vitro and in post-metaphase cell lysates with BAF. Subfractions of BAF relocalize to LAP2 $\alpha$ labeled structures during nuclear reassembly after mitosis. Thus, LAP2 $\alpha$ and BAF seem to cooperate with lamins in the reorganization of telomeres and overall chromatin after cell division [34].

Binding of lamins to telomeres is mediated at least partly by the shelterin factor TRF2. The lamin A-TRF2 association seems to be important not only for maintaining the typical protective T-loops at telomeres, but also for stabilizing the formation of T-loops with interstitial telomeric sequences (ITSs)[35]. These consist of tandem telomeric repeats localized at intrachromosomal sites that can form T-loops, known as interstitial T-loops (ITLs). Lamin A/C reduction or progerin expression lead to fewer ITLs and profound telomere loss. Importantly, overexpression of mutant and wild-type lamin A in human fibroblasts results in accelerated telomere shortening and replicative senescence[36], supporting the notion that alterations in A-type lamins disrupt telomere homeostasis. Consistently, HGPS patient-derived fibroblasts exhibit telomere shortening during proliferation in culture, which accompanies premature entry into 
senescence[37,38]. Telomerase expression or p53 inactivation suppresses the proliferative defects[39], suggesting that telomere dysfunction and activation of checkpoints contribute to these defects. The current view is that alterations in telomere structure/length due to disruption of the nuclear lamina activate the DNA damage response, which in turn activates checkpoints and p53-dependent growth arrest. Moreover, studies in $\mathrm{Lmna}^{-/-}$mouse embryonic fibroblasts (MEFs) reveal a modest but consistent decrease in telomere length, as well as an increase in damaged telomeres, compared to $L m n a^{++}$MEFs. This provides further evidence for the role of lamins in maintaining telomere homeostasis[13].

Molecular mechanisms behind lamin-directed telomere attrition remain ill defined. Telomerase activity does not decrease in lamin A/C-deficient cells. Similarly, recruitment of shelterin components TRF1 and TRF2 to telomeres is not altered. Moreover, there is no increase in recombination at telomeres, which could explain the loss of telomere sequence[13]. However, telomere recruitment of telomerase may be hindered due to loss of telomere tethering to the lamin meshwork, leading to telomere shortening. Lamin $\mathrm{A} / \mathrm{C}$ deficiency is also associated with DNA replication defects. Thus loss of telomere tethering could contribute to telomere replication defects, and in turn to progressive telomere shortening. Moreover, telomeric chromatin exhibits a mix of heterochromatic and euchromatic histone marks, known to affect telomere length control[5-7]. The fact that lamin A/C deficiency results in epigenetic changes at telomeres[13] suggests that alterations in telomere epigenetics could impact telomere homeostasis.

In addition to the role of lamins in telomere length maintenance, studies have shown the indirect participation of lamins in the aberrant processing of dysfunctional telomeres by NHEJ[13,40,41]. In particular, expression of a TRF2 dominant-negative mutant (TRF2 $\left.{ }^{\triangle \mathrm{B} \Delta \mathrm{M}}\right)$ in lamin A/C-proficient cells leads to telomere uncapping and massive end-to-end fusions. In contrast, lamin A/C-deficient cells exhibit markedly reduced NHEJ of dysfunctional telomeres. 
This deficiency is linked to 53BP1 degradation, an essential protein for this process[20]. As such, stabilization of 53BP1 in the context of lamin A/C-deficiency restores NHEJ of dysfunctional telomeres induced by $\mathrm{TRF}^{\mathrm{\Delta B} \Delta \mathrm{M}}$ expression[13,40,41]. These studies suggest that the role of lamins in NHEJ of dysfunctional telomeres is independent of their structural role tethering telomeres. Rather, loss of lamin A/C affects NHEJ indirectly by reducing the levels of 53BP1. Altogether, these studies provide strong evidence for alterations in lamins leading to telomere dysfunction-driven genomic instability.

\section{Reciprocal relationship between telomere dysfunction and progerin production}

The excessive telomere attrition in HGPS patient-derived fibroblasts is linked to the expression of progerin, the mutant protein that results from aberrant splicing and defective processing of lamin A. As such, hematopoietic cells from these patients, which typically do not express lamin A/progerin, do not exhibit telomere shortening[38]. Interestingly, ectopic expression of telomerase ameliorates a variety of HGPS cellular phenotypes [39]. Telomerase expression allows senescence bypass by decreasing DNA damage signaling and thus preventing the activation of p53 and Rb pathways[42], which mediate the onset of premature senescence. While telomerase does not reduce progerin expression, it is protective against DNA damage accumulation at telomeres. This provides strong evidence for progerin expression-induced senescence implicating telomere dysfunction. Moreover, a recent study using doxycycline-induced progerin expression in normal fibroblasts was able to recapitulate HGPS cellular phenotypes in a dose-dependent manner, including DNA damage accumulation, proliferation defects, and telomere attrition[33]. Ectopic telomerase expression ameliorated these phenotypes, in addition to progerin-induced changes in gene expression. In particular, the expression of 142 genes was significantly altered in progerin-expressing cells, many of them associated with senescence. Nearly all these changes in gene expression were prevented by 
telomerase [33]. Moreover, this study showed that ESCs, which express high levels of telomerase, are protected from progerin-induced phenotypes. All these data indicate that telomere dysfunction caused by progerin production contributes to a variety of HGPS cellular phenotypes, and that telomerase protects from HGPS cellular decline.

In addition to progerin causing telomere dysfunction, studies have shown that telomere dysfunction causes progerin accumulation. For example, normal human fibroblasts undergoing replicative senescence exhibit elevated progerin production, while this was not observed during telomere-independent cellular senescence[43]. Increased progerin transcripts and protein levels are also observed in response to telomere dysfunction induced by $T R F 2^{\Delta B \Delta M}$ expression[43]. Elevated progerin production seems to be due to changes in alternative splicing in response to telomere damage; indeed a variety of genes are alternatively spliced during replicative senescence. Importantly, bypass of replicative senescence in normal fibroblasts by telomerase overexpression inhibits activation of the cryptic splice site that results in progerin production.

Together, these data suggest a reciprocal disastrous relationship between telomere dysfunction and progerin production, such that progerin production hinders telomere maintenance, as shown in HGPS fibroblasts, and telomere dysfunction activates progerin production, as shown during replicative senescence in normal fibroblasts. This study provides a molecular mechanism by which cells from old individuals activate progerin production, as well as how progerin expression promotes physiological aging. Thus, telomere homeostasis during aging is critical not only for the maintenance of genome stability, but also for proper control of alternative splicing, which in the case of the LMNA gene can be highly detrimental at the cellular and organismal level. Intriguingly, recent studies have shown that alterations in splicing can directly affect telomere biology. In particular, Listerman et al. identified splicing factors that regulate the expression of splice variants of human telomerase. For instance, overexpression of SRSF11, a member of the highly conserved family of serine/arginine (SR) proteins required for 
constitutive pre-mRNA splicing, induces the expression of a dominant negative variant of telomerase[44]. This suggests that alterations in the splicing machinery can cause telomere dysfunction. More recently, Lee et al showed binding of SRSF11 to telomeres through TRF2 and to telomerase via TERC[45]. They also showed that SRSF11 is required for telomerase recruitment to telomeres during $S$ phase and for telomere elongation. As such, SRSF11 depletion leads to progressive telomere shortening during proliferation[45]. Although additional mechanistic studies are needed to understand how splicing factors such as SRSF11 regulate telomerase recruitment and telomere homeostasis, it is tempting to speculate that progerin expression disrupts the function of splicing factors, which in turn could result in telomere attrition/dysfunction.

\section{Role of lamins in telomere compartmentalization and movement}

Compartmentalization of the genome in the 3D nuclear space has emerged as an important factor in the maintenance of genome function and integrity. Sophisticated high-throughput techniques have been developed for mapping chromatin contacts across different genomic distances and between chromosomes. These have revealed that the genome is organized into topologically associating domains (TADs). TADs are defined by high levels of chromatin interactions within the region, interspersed with regions of little or no interaction with neighboring regions[9]. The central domains of TADs are enriched in tissue-specific genes and their enhancers, while the border regions between TADs are enriched in housekeeping genes. In addition to TADs, the tethering of chromatin to nuclear subcompartments such as the lamina and proteinaceous nuclear bodies represents another level of higher-order genome organization[9,10,22]. Large genomic domains have been found to associate with the nuclear lamina using techniques such as DNA adenine methyltransferase identification (DamID) and ChIP-seq (chromatin immunoprecipitation followed by sequencing) (Figure 2)[23,46]. These 
lamina-associated domains (LADs) range in size between 0.1 and 10 megabases, covering nearly $40 \%$ of the genome. Most genes in LADs are expressed at low levels and are enriched in repressive chromatin marks. LADs are considered units of chromosome organization within the nucleus that are demarcated by the insulator protein CTCF, by promoters oriented away from LADs, or by $\mathrm{CpG}$ islands. Interestingly, LADs have been mapped to some subtelomeric domains[46], and suggested to target their proximal telomere to the nuclear envelope. One such example is the macrosatellite D4Z4 repeat located at 4q35 subtelomeric locus, which helps localize its proximal telomere to the nuclear periphery. The role of $D 4 Z 4$ repeat element as a cis-acting positioning element depends on CTCF and lamin A/C binding[47]. These findings reinforce a role for lamins in the subnuclear distribution of telomeres. In support of this notion, ChIP experiments show association of telomeric sequences with lamin A/C. Intriguingly, studies in lamin A/C-deficient cells, i.e. Lmna ${ }^{-/}$MEFs, revealed that A-type lamins play a role in the distribution of telomeres throughout the entire nucleoplasm, and not only in the accumulation at the nuclear periphery. In particular, an increased accumulation of telomeres at the nuclear periphery and away from the nuclear interior was observed in Lmna ${ }^{-/-}$MEFs, when compared to $\mathrm{Lmna}^{+/+}$counterparts[13]. A model was proposed whereby the nuclear periphery represents the default pathway for telomere localization, potentially via association of telomeres with B-type lamins and/or lamina-associated proteins. In this model, A-type lamins in the nuclear interior would be responsible for the distribution of telomeres throughout the nucleoplasm. Interestingly, the altered localization of telomeres in $L m n a^{-/}$MEFs is accompanied by telomere attrition or loss. Whether the altered distribution of telomeres in lamin A/C-deficient cells contributes to telomere dysfunction remains to be demonstrated directly.

In addition to telomere compartmentalization, lamins impact telomere mobility within the nucleus. Live microscopy studies have shown that chromatin motion in the nucleus has highly constrained dynamics. Measuring individual trajectories of fluorescently labeled telomeres 
revealed that their motion is not random. Rather, telomere motion is slowed-down by the environment, being characterized as anomalous diffusion[48]. The same anomalous diffusion characterizes other genomic sites such as centromeres and unique gene loci. Interestingly, Lmna $^{-/}$MEFs exhibit a dramatic change in telomere dynamics, with a transition from slow anomalous diffusion to fast and normal diffusion[48]. Thus, lamin A/C deficiency results in much faster dynamics, as well as larger areas scanned by each individual telomere (Figure 3). Reconstitution of lamin $A$ in lamin A/C-deficient cells fully recovers the slow dynamics of telomeres[48]. In contrast, expression of mutants in the rod or tail domain of the lamin A protein only partially corrected the slow telomere dynamics. Consistent with these findings, fibroblasts from a patient homozygous for a nonsense Y259X mutation in the LMNA gene that are devoid of lamin A/C expression exhibited hypermobility of telomeres[49]. In contrast, HGPS fibroblasts showed an overall reduction in telomere dynamics compared to normal fibroblasts[49]. The opposite dynamic behavior of telomeres in the absence of lamin A/C or upon expression of progerin reveals potential differences in telomere tethering to lamins in both contexts. Increased mobility upon lamin loss could be explained by loss of tethering, and the decreased mobility in progerin-expressing cells could be the result of increased tethering. However, the picture seems to be more complex, as pull-down assays showed that TRF2, the shelterin component thought to mediate telomere binding to lamins, has reduced ability to bind progerin[35]. This suggests that telomere tethering to lamins could be reduced in HGPS cells. These conflicting data stress the need of additional studies to characterize the mechanism of telomeres tethering to lamins. For instance, depletion/reconstitution experiments of different shelterin components and lamin A/C mutants (i.e. progerin) followed by telomere chromatin immunoprecipitation and telomere mobility studies would provide detailed insight about the players involved and their specific roles. Overall, these studies indicate that disruptions of the nuclear lamina due to mutation or reduced expression of lamins have dramatic effects on telomere mobility (Figure 3). Lamins 
seem to act as a scaffold that restrains chromatin movement. The absence of this restraining structure allows genomic loci to undergo large displacements over time.

Interestingly, a recent study also implicated components of the nuclear envelope, such as the linker of the nucleoskeleton, and cytoskeleton (LINC) complex (formed by SUN1/2 proteins and Nesprins), and microtubule motor proteins (Kinesins), in the mobility of dysfunctional telomeres, as well as of DNA DSBs within the nucleus. In addition, 53BP1/LINC/microtubuledependent movement is important for NHEJ of dysfunctional telomeres and DSBs[50]. All together, these studies indicate that damaged DNA/chromatin exhibits increased mobility within the nucleus, and that this mobility is critical for its proper repair. The DNA repair factor 53BP1, LINC-microtubule connections, and the lamin network, all seem to play a role in these processes. Characterizing how these connections are established and regulated will be important to a mechanistic understanding of how nuclear architecture impacts genome stability.

\section{Conclusions and perspectives}

Telomere function is critical for maintenance of genome stability and cell viability. Telomere dysfunction can arise due to excessive attrition of telomeric repeats during DNA replication or due to loss of the capping protective T-loop structure. Similarly, loss of heterochromatic marks or telomeric transcripts (TERRAs) hinders telomere length homeostasis, in part by activating recombination among telomeric repeats. Recent studies uncovered functional interactions between telomeres and lamins, highlighting lamins as an example of nuclear architecture regulating genome function. The fact that alterations in lamins result in telomere attrition, changes in telomere chromatin modifications and transcription, and increased telomere damage, suggests that the scaffolding role of lamins could represent a distinct level of telomere regulation. The basis for this regulation could be that telomeres tethering to lamins ensures telomere compartmentalization and constrained mobility, which are key for the accessibility 
and/or recruitment of the proteins that are required for the maintenance of functional telomeres: telomerase, shelterin complex, chromatin-modifying activities, DNA repair factors, RNA polymerase, etc. However, we cannot exclude that the role of lamins in telomere biology is independent of their nuclear structural role. In fact, alterations in lamins due to overexpression, depletion, or mutation result in profound changes in global gene expression, as well as changes in protein degradation pathways. Thus, the telomere defects in lamin-deficient cells could be the indirect result of changes in gene expression and/or protein stability. To address these questions will require detailed characterization of the process of telomere tethering to nuclear lamins, identifying telomere-binding factors that mediate the tethering or untethering, how this tethering process is regulated during cell cycle and cell division, and engineering separation-offunction lamin mutant proteins to pinpoint the different roles of lamins in telomere biology. 


\section{Acknowledgements}

Research in the Gonzalo laboratory was supported by the NIH (RO1 GM094513-01), DOD BCRP Idea Award BC110089, Presidential Research Award from Saint Louis University, and the Siteman Cancer Center Research Development Award. Authors declare no conflict of interest. We acknowledge Dr. Yuval Garini for sharing unpublished images of telomere mobility to include in Figure 3. 
References and recommended reading:

Recent papers of particular interest have been highlighted as: *of special interest; **of outstanding interest.

1. Schmidt JC, Cech TR: Human telomerase: biogenesis, trafficking, recruitment, and activation. Genes Dev 2015, 29:1095-1105.

2. Doksani $\mathrm{Y}$, de Lange $\mathrm{T}$ : The role of double-strand break repair pathways at functional and dysfunctional telomeres. Cold Spring Harb Perspect Biol 2014, 6:a016576.

3. Martinez P, Blasco MA: Replicating through telomeres: a means to an end. Trends Biochem Sci 2015, 40:504-515.

4. Cusanelli E, Chartrand P: Telomeric repeat-containing RNA TERRA: a noncoding RNA connecting telomere biology to genome integrity. Front Genet 2015, 6:143.

5. Benetti R, Gonzalo S, Jaco I, Schotta G, Klatt P, Jenuwein T, Blasco MA: Suv4-20h deficiency results in telomere elongation and derepression of telomere recombination. J Cell Biol 2007, 178:925-936.

6. Gonzalo S, Garcia-Cao M, Fraga MF, Schotta G, Peters AH, Cotter SE, Eguia R, Dean DC, Esteller $M$, Jenuwein $T$, et al:: Role of the RB1 family in stabilizing histone methylation at constitutive heterochromatin. Nat Cell Biol 2005, 7:420-428.

7. Gonzalo S, Jaco I, Fraga MF, Chen T, Li E, Esteller M, Blasco MA: DNA methyltransferases control telomere length and telomere recombination in mammalian cells. Nat Cell Biol 2006, 8:416-424.

8. Wang Z, Deng Z, Dahmane N, Tsai K, Wang P, Williams DR, Kossenkov AV, Showe LC, Zhang R, Huang Q, et al.: Telomeric repeat-containing RNA (TERRA) constitutes a nucleoprotein component of extracellular inflammatory exosomes. Proc Natl Acad Sci U S A 2015, 112:E6293-6300. 
9. Pombo A, Dillon N: Three-dimensional genome architecture: players and mechanisms. Nat Rev Mol Cell Biol 2015, 16:245-257.

${ }^{* *}$ Excellent review about the current view of cellular factors that contribute to the compartmentalization of the genome in the 3D nuclear space, as well as the formation of higherorder chromatin structures. In addition, it summarizes current knowledge about how genome architecture influences genome function and cellular processes such as cell cycle, differentiation, and senescence.

10. Mattout A, Cabianca DS, Gasser SM: Chromatin states and nuclear organization in development--a view from the nuclear lamina. Genome Biol 2015, 16:174.

11. Bickmore WA, van Steensel B: Genome architecture: domain organization of interphase chromosomes. Cell 2013, 152:1270-1284.

12. Solovei I, Wang AS, Thanisch K, Schmidt CS, Krebs S, Zwerger M, Cohen TV, Devys D, Foisner $R$, Peichl $L$, et al.: LBR and lamin A/C sequentially tether peripheral heterochromatin and inversely regulate differentiation. Cell 2013, 152:584-598.

13. Gonzalez-Suarez I, Redwood AB, Perkins SM, Vermolen B, Lichtensztejin D, Grotsky DA, Morgado-Palacin L, Gapud EJ, Sleckman BP, Sullivan T, et al.: Novel roles for A-type lamins in telomere biology and the DNA damage response pathway. EMBO J 2009, 28:2414-2427.

*This study provided a comprehensive analysis of the types of genomic instability observed in mouse embryonic fibroblasts from Lmna knockout mice. In particular, alterations in the nuclear localization of telomeres, telomere shortening, and defects in DNA repair by NHEJ are reported. 14. Arnoult N, Schluth-Bolard C, Letessier A, Drascovic I, Bouarich-Bourimi R, Campisi J, Kim SH, Boussouar A, Ottaviani A, Magdinier F, et al.: Replication timing of human telomeres is chromosome arm-specific, influenced by subtelomeric structures and connected to nuclear localization. PLoS Genet 2010, 6:e1000920. 
15. Guidi M, Ruault M, Marbouty M, Loiodice I, Cournac A, Billaudeau C, Hocher A, Mozziconacci J, Koszul R, Taddei A: Spatial reorganization of telomeres in longlived quiescent cells. Genome Biol 2015, 16:206.

16. Barutcu AR, Lajoie BR, McCord RP, Tye CE, Hong D, Messier TL, Browne G, van Wijnen AJ, Lian JB, Stein JL, et al.: Chromatin interaction analysis reveals changes in small chromosome and telomere clustering between epithelial and breast cancer cells. Genome Biol 2015, 16:214.

17. Lee CY, Horn HF, Stewart CL, Burke B, Bolcun-Filas E, Schimenti JC, Dresser ME, Pezza $\mathrm{RJ}$ : Mechanism and regulation of rapid telomere prophase movements in mouse meiotic chromosomes. Cell Rep 2015, 11:551-563.

18. Wang X, Kam Z, Carlton PM, Xu L, Sedat JW, Blackburn EH: Rapid telomere motions in live human cells analyzed by highly time-resolved microscopy. Epigenetics Chromatin 2008, 1:4.

19. Makhija E, Jokhun DS, Shivashankar GV: Nuclear deformability and telomere dynamics are regulated by cell geometric constraints. Proc Natl Acad Sci U S A 2016, 113:E32-40.

20. Dimitrova N, Chen YC, Spector DL, de Lange T: 53BP1 promotes non-homologous end joining of telomeres by increasing chromatin mobility. Nature 2008, 456:524-528.

${ }^{\star *}$ This study demonstrated that functional telomeres are somewhat constrained within the nucleus, while dysfunctional telomeres exhibit markedly increased mobility. It also showed that phosphorylation of ATM and the presence 53BP1 are criticial for the increased mobility of dysfunctional telomeres.

21. Swift J, Ivanovska IL, Buxboim A, Harada T, Dingal PC, Pinter J, Pajerowski JD, Spinler KR, Shin JW, Tewari M, et al.: Nuclear lamin-A scales with tissue stiffness and enhances matrix-directed differentiation. Science 2013, 341:1240104. 
* Study reveals that the levels of nuclear lamin A scales with tissue elasticity, similar to levels of collagens in the extracellular matrix, and that tissue stiffness and stress increase lamin A expression. In addition, the study shows that stem cell differentiation into fat on soft matrix was promoted by reduced lamin A levels, while differentiation into bone on stiff matrix was promoted by increased lamin A. Moreover, the study reveals that the vitamin A/retinoic acid (RA) pathway regulates expression of the LMNA gene, while expression of lamin A regulates the entry of RA receptors into the nucleus.

22. Gay S, Foiani M: Nuclear envelope and chromatin, lock and key of genome integrity. Int Rev Cell Mol Biol 2015, 317:267-330.

23. Amendola $M$, van Steensel $B$ : Mechanisms and dynamics of nuclear lamina-genome interactions. Curr Opin Cell Biol 2014, 28:61-68.

24. Bonello-Palot N, Simoncini S, Robert S, Bourgeois P, Sabatier F, Levy N, Dignat-George F, Badens C: Prelamin A accumulation in endothelial cells induces premature senescence and functional impairment. Atherosclerosis 2014, 237:45-52.

25. Moiseeva O, Lessard F, Acevedo-Aquino M, Vernier M, Tsantrizos YS, Ferbeyre G: Mutant lamin A links prophase to a p53 independent senescence program. Cell Cycle 2015, 14:2408-2421.

26. Schreiber KH, Kennedy BK: When Lamins Go Bad: Nuclear Structure and Disease. Cell 2013, 152:1365-1375.

27. Merideth MA, Gordon LB, Clauss S, Sachdev V, Smith AC, Perry MB, Brewer CC, Zalewski C, Kim HJ, Solomon B, et al.: Phenotype and course of Hutchinson-Gilford progeria syndrome. N Engl J Med 2008, 358:592-604.

${ }^{\star *}$ This landmark study is a comprehensive clinical investigation of HGPS in 15 children between 1 and 17 years of age. The study explains in detail the pathophysiology of the disease, which includes growth impairment, sclerotic skin, joint contractures, bone abnormalities, nervous system sequelae, audiological defects, oral deficits, prolonged prothrombin times, elevated 
platelet counts and serum phosphorus levels, and cardiovascular disease characterized by high blood pressure, reduced vascular compliance, decreased ankle-brachial indexes, and adventitial thickening.

28. Scaffidi P, Misteli T: Lamin A-dependent nuclear defects in human aging. Science 2006, 312:1059-1063.

29. Gonzalo S: DNA damage and lamins. Adv Exp Med Biol 2014, 773:377-399.

30. Gonzalo S, Kreienkamp R: DNA repair defects and genome instability in HutchinsonGilford Progeria Syndrome. Curr Opin Cell Biol 2015, 34:75-83.

31. Shoeman $R L$, Traub $P$ : The in vitro DNA-binding properties of purified nuclear lamin proteins and vimentin. $J$ Biol Chem 1990, 265:9055-9061.

32. Dechat T, Gajewski A, Korbei B, Gerlich D, Daigle N, Haraguchi T, Furukawa K, Ellenberg J, Foisner R: LAP2alpha and BAF transiently localize to telomeres and specific regions on chromatin during nuclear assembly. J Cell Sci 2004, 117:6117-6128.

33. Chojnowski A, Ong PF, Wong ES, Lim JS, Mutalif RA, Navasankari R, Dutta B, Yang H, Liow YY, Sze SK, et al.: Progerin reduces LAP2alpha-telomere association in Hutchinson-Gilford progeria. Elife 2015, 4.

*This recent report shows a dose-dependent effect of progerin expression in normal fibroblasts inducing cellular phenotypes characteristic of HGPS patient-derived fibroblasts. This finding suggest that reducing progerin levels under a threshold would be sufficient to ameliorate the HGPS phenotype. Importantly, it also shows that progerin expression induces changes in gene expression, which are reduced by telomerase expression.

34. Loi M, Cenni V, Duchi S, Squarzoni S, Lopez-Otin C, Foisner R, Lattanzi G, Capanni C: Barrier-to-Autointegration Factor (BAF) involvement in prelamin A-related chromatin organization changes. Oncotarget 2015. 
35. Wood AM, Rendtlew Danielsen JM, Lucas CA, Rice EL, Scalzo D, Shimi T, Goldman RD, Smith ED, Le Beau MM, Kosak ST: TRF2 and lamin A/C interact to facilitate the functional organization of chromosome ends. Nat Commun 2014, 5:5467.

${ }^{*}$ Authors present evidence that T-loops form at interstitial telomere sequences in a TRF2dependent and lamin A/C-dependent manner. Reduced levels of lamin A/C or mutations in the LMNA gene that cause HGPS lead to reduced interstitial T-loop formation and telomere loss. These findings provide a mechanism behind telomere dysfunction in cells with a disrupted nuclear lamina.

36. Huang S, Risques RA, Martin GM, Rabinovitch PS, Oshima J: Accelerated telomere shortening and replicative senescence in human fibroblasts overexpressing mutant and wild-type lamin A. Exp Cell Res 2008, 314:82-91.

37. Allsopp RC, Vaziri H, Patterson C, Goldstein S, Younglai EV, Futcher AB, Greider CW, Harley CB: Telomere length predicts replicative capacity of human fibroblasts. Proc Natl Acad Sci U S A 1992, 89:10114-10118.

38. Decker ML, Chavez E, Vulto I, Lansdorp PM: Telomere length in Hutchinson-Gilford progeria syndrome. Mech Ageing Dev 2009, 130:377-383.

39. Kudlow BA, Stanfel MN, Burtner CR, Johnston ED, Kennedy BK: Suppression of proliferative defects associated with processing-defective lamin A mutants by hTERT or inactivation of p53. Mol Biol Cell 2008, 19:5238-5248.

${ }^{* *}$ First study to demonstrate that progerin interferes with telomere structure and homeostasis, leading to premature entry into senescence. Importantly, the study demonstrates that ectopic expression of telomerase suppresses the early proliferative defects associated with progerin expression, suggesting that telomere dysfunction plays a role in the celluar decline characteristic of HGPS patient-derived cells. 
40. Gonzalez-Suarez I, Redwood AB, Grotsky DA, Neumann MA, Cheng EH, Stewart CL, Dusso A, Gonzalo S: A new pathway that regulates 53BP1 stability implicates cathepsin L and vitamin D in DNA repair. EMBO J 2011, 30:3383-3396.

41. Redwood AB, Perkins SM, Vanderwaal RP, Feng Z, Biehl KJ, Gonzalez-Suarez I, MorgadoPalacin L, Shi W, Sage J, Roti-Roti JL, et al.: A dual role for A-type lamins in DNA double-strand break repair. Cell Cycle 2011, 10:2549-2560.

42. Benson EK, Lee SW, Aaronson SA: Role of progerin-induced telomere dysfunction in HGPS premature cellular senescence. J Cell Sci 2010, 123:2605-2612.

43. Cao K, Blair CD, Faddah DA, Kieckhaefer JE, Olive M, Erdos MR, Nabel EG, Collins FS: Progerin and telomere dysfunction collaborate to trigger cellular senescence in normal human fibroblasts. J Clin Invest 2011, 121:2833-2844.

${ }^{* *}$ Interesting study showing that progressive telomere damage during senescence in human fibroblasts causes activation of progerin production. Progressive telomere damage seems to affect alternative splicing of a variety of genes. These findings provided some clues about how progerin expression might participate in the normal aging process.

44. Listerman I, Sun J, Gazzaniga FS, Lukas JL, Blackburn EH: The major reverse transcriptase-incompetent splice variant of the human telomerase protein inhibits telomerase activity but protects from apoptosis. Cancer Res 2013, 73:2817-2828.

45. Lee JH, Jeong SA, Khadka P, Hong J, Chung IK: Involvement of SRSF11 in cell cyclespecific recruitment of telomerase to telomeres at nuclear speckles. Nucleic Acids Res 2015 .

*The authors show for the first time a role for splicing factors in telomere biology. The splicing factor SRSF11 is a TERC- and TRF2-binding protein that helps recruit telomerase and telomeres at nuclear speckles during $S$ phase of the cell cycle. SRSF11 loss abrogates telomere elongation by telomerase. 
46. Guelen L, Pagie L, Brasset E, Meuleman W, Faza MB, Talhout W, Eussen BH, de Klein A, Wessels L, de Laat W, et al.: Domain organization of human chromosomes revealed by mapping of nuclear lamina interactions. Nature $2008,453: 948-951$.

47. Ottaviani A, Schluth-Bolard C, Rival-Gervier S, Boussouar A, Rondier D, Foerster AM, Morere J, Bauwens S, Gazzo S, Callet-Bauchu E, et al.: Identification of a perinuclear positioning element in human subtelomeres that requires A-type lamins and CTCF. Embo J 2009, 28:2428-2436.

48. Bronshtein I, Kepten E, Kanter I, Berezin S, Lindner M, Redwood AB, Mai S, Gonzalo S, Foisner $\mathrm{R}$, Shav-Tal $\mathrm{Y}$, et al.: Loss of lamin A function increases chromatin dynamics in the nuclear interior. Nat Commun 2015, 6:8044.

${ }^{* *}$ The authors use fluorescently labeled telomeres and measure diffusion of telomeres in live cells. The study finds that telomeres show highly constrained dynamics due to tethering to lamins. Loss of lamin A leads to a dramatic transition from slow anomalous diffusion to fast and normal diffusion, as well as greatly enhanced volume of telomere motion.

49. De Vos WH, Houben F, Hoebe RA, Hennekam R, van Engelen B, Manders EM, Ramaekers FC, Broers JL, Van Oostveldt P: Increased plasticity of the nuclear envelope and hypermobility of telomeres due to the loss of A-type lamins. Biochim Biophys Acta 2010, 1800:448-458.

50. Lottersberger F, Karssemeijer RA, Dimitrova N, de Lange T: 53BP1 and the LINC Complex Promote Microtubule-Dependent DSB Mobility and DNA Repair. Cell 2015, 163:880893.

${ }^{* *}$ Study shows that damaged chromatin, such as dysfunctional telomeres or IR-induced DNA double strand-breaks (DSBs), exhibits greater mobility in the nuclear space. This enhanced mobility requires 53BP1, the LINC complex, and dynamic microtubules, and is important for the repair of damaged DNA/chromatin. However, in the context of BRCA1 deficiency, increased mobility causes mis-rejoining of drug-induced DSBs and increased genomic instabiity. 


\section{Figure legends}

Figure 1. Structure of telomeres. (A) Telomeres are nucleoprotein structures that protect the ends of eukaryotic chromosomes. In vertebrates, telomeres consist of long arrays of doublestranded DNA (dsDNA) repeats of the [TTAGGG]n sequence, ending in a single 3' G-strand overhang. Telomeric dsDNA repeats are bound by a specialized protein complex known as shelterin, which includes TRF1 (red), TRF2 (beige), TIN2 (dark blue), RAP1 (light blue), POT1 (yellow), and TPP1 (brown) proteins. Telomerase is a ribonucleoprotein complex with reverse transcriptase activity that adds telomeric repeats after DNA replication using the G-strand overhang as substrate. (B) Telomeres form a capping structure in which the 3' G-strand overhang invades the dsDNA region forming a T (telomere)-loop. The displaced strand forms a D (displacement)-loop. This capping structure protects chromosome from erosion.

Figure 2. Nuclear distribution of lamins, lamin-associated proteins, and telomeres. Diagram depicting the proposed localization of lamins at the nuclear lamina and extending into the nucleoplasm. Lamins associate with a variety of proteins at the nuclear periphery, including emerin, SUN1/2 proteins, lamin B receptor (LBR), lamin-associated proteins (LAPs), barrier-toautointegration factor (BAF), as well as nuclear pore complexes (NPCs), among others. Lamins have the ability to bind DNA and chromatin, and chromosomal domains with specific characteristics have been shown to associate with lamins (LADs), including telomeres. Lamins are viewed as a scaffold for compartmentalization of the genome in the 3D nuclear space, with implications for genome function and stability. Alterations in lamins result in changes in telomere localization, dynamics, structure, length, and function. 
Figure 3. Telomere dynamics. (A) Image shows overall telomere trajectories in one nucleus of U2OS cells expressing GFP-TRF1. Each green oval highlighted within the larger green nucleus represents the domain occupied by a single telomere. Full 3D images were captured every 25 seconds with a confocal microscope to measure telomere dynamics. Multicolor paths extending from each green oval represent the reconstructed motion of that telomere as determined by IMARIS, a 3D/4D image processing software. Approximately 100 time points are observed for each telomere. This random motion can be further analyzed in order to extract the actual type of the diffusion motion. (B) Magnification of a 3D image to highlight the multi-colored mobility tracts of a few telomeres (green ovals) within a single nucleus. (C) The area scanned by telomeres in MEFs $\mathrm{Lmna}^{+/+}$as calculated from the motion of each telomere during 35 minutes. Data were measured in $3 \mathrm{D}$ with a confocal microscope, and the projection images were calculated. The color is an indication of the scanned volume size, with the scaled heat map colors shown to the right of the panel. Each trapezoid represents the extent of the nuclear volume traversed by a single telomere during the experiment. Each image shows the projection of telomeres measured from $15-20$ cells $(\approx 200$ telomeres are shown). (D) Area scanned by telomeres $(\approx 200$ telomeres shown) in Lmna ${ }^{-/}$MEFs calculated as in (C). Note the dramatically expanded trapezoid areas and larger scale values compared to $(\mathrm{C})$. 
A
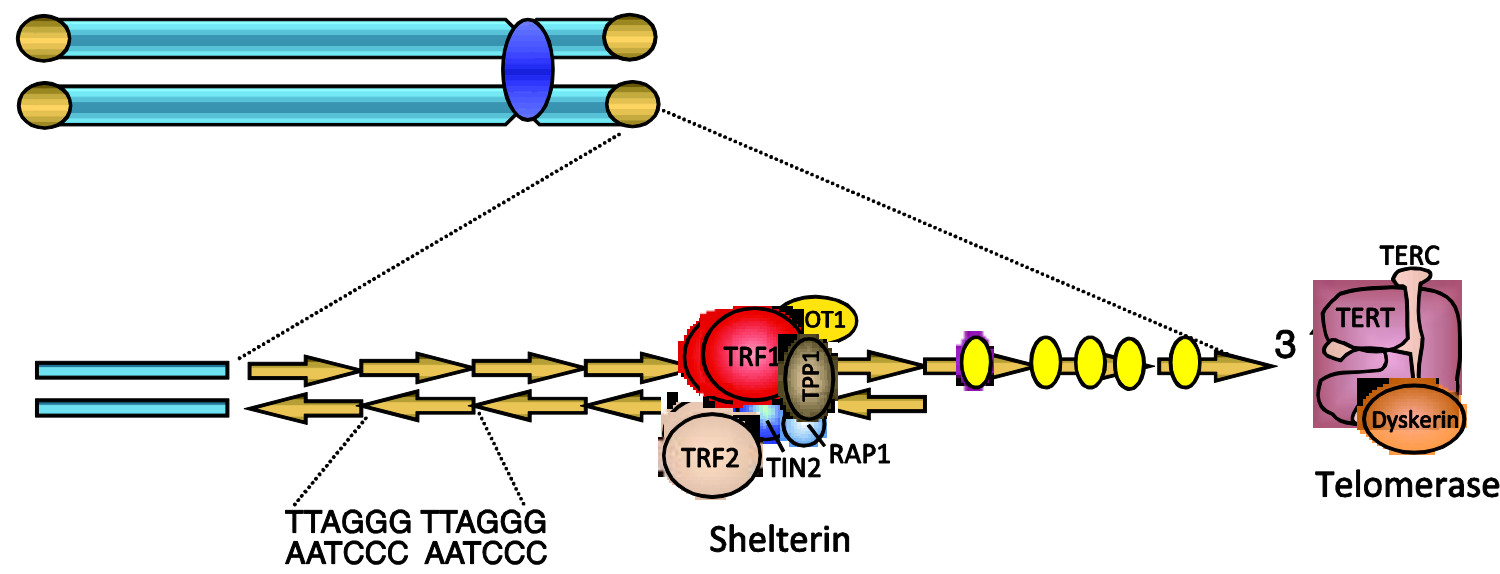

TTAGGG TTAGGG
AATCCC AATCCC

Shelterin

B

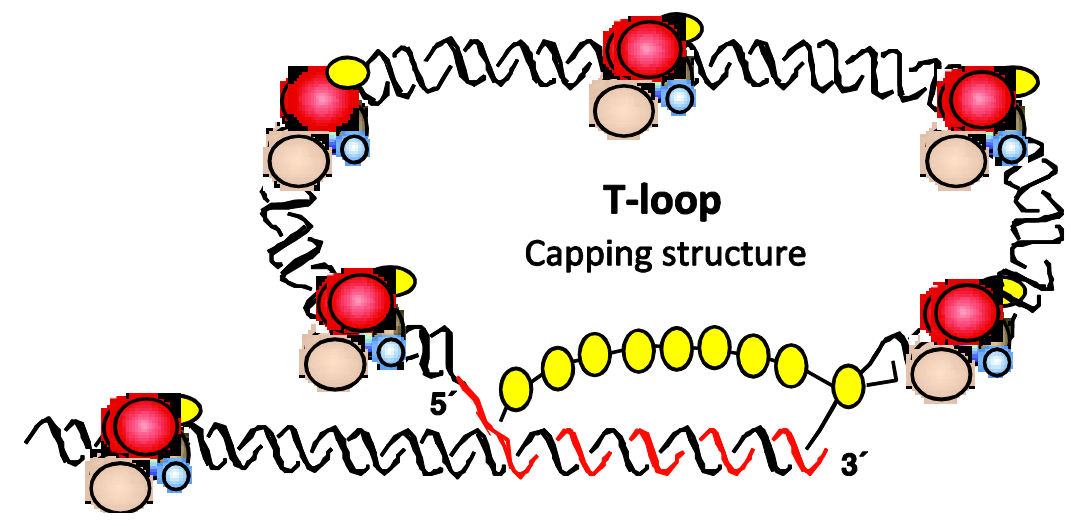




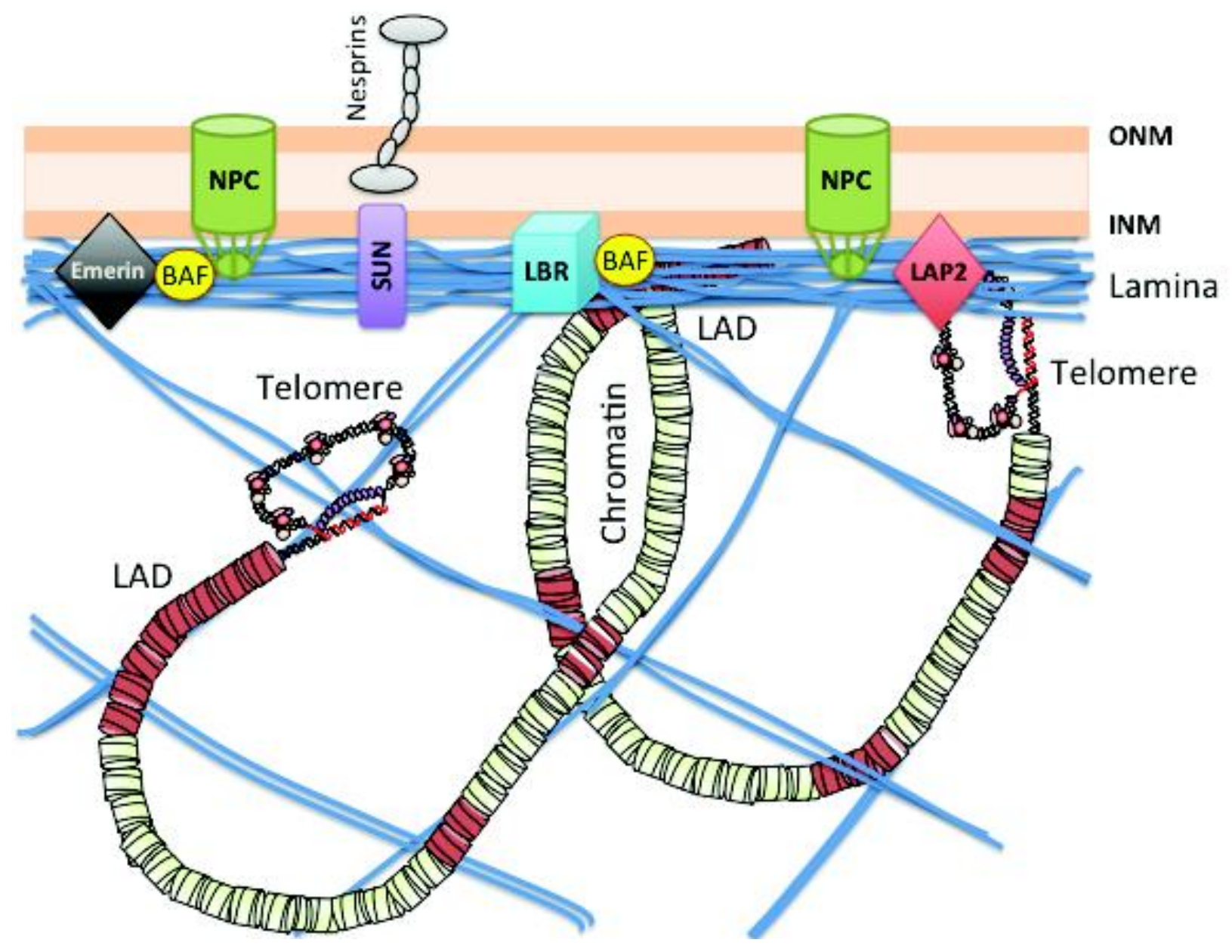


A

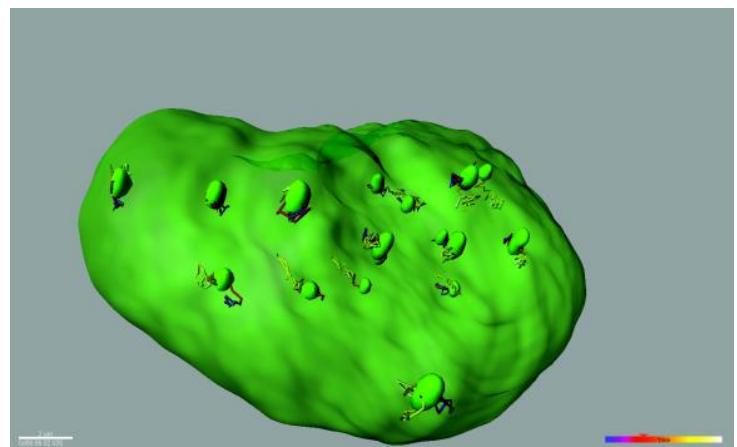

C

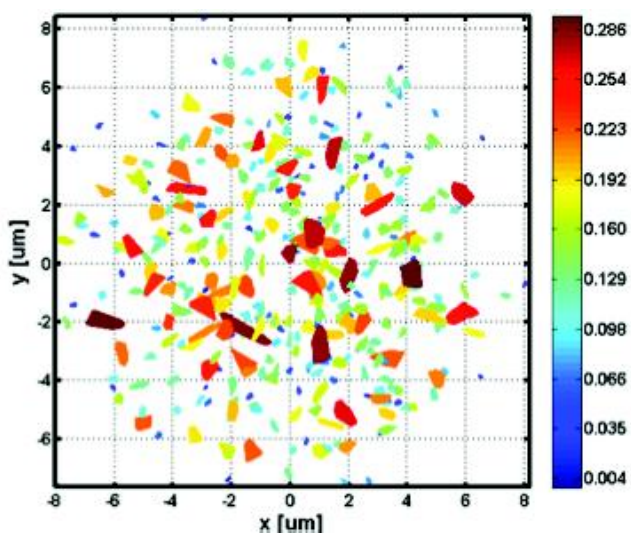

B

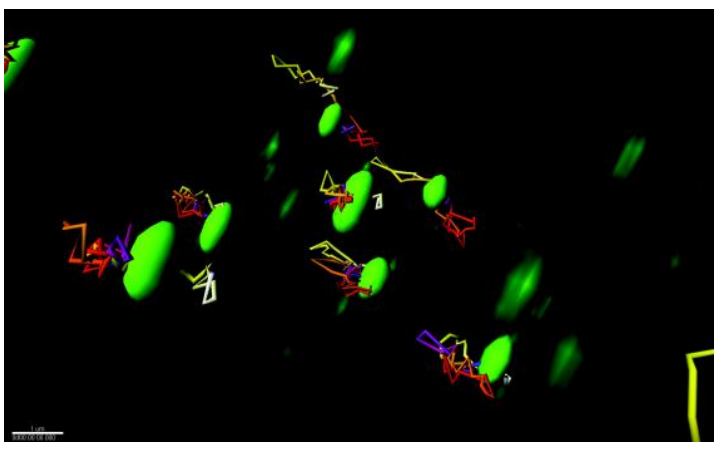

D

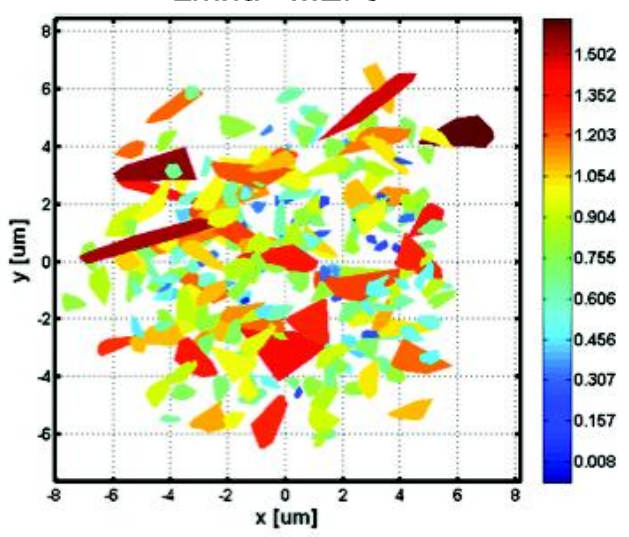

